\title{
PARALISIA CEREBRAL DIAGNÓSTICO ETIOLÓGICO
}

\author{
CEREBRAL PALSY AETIOLOGIC DIAGNOSIS
}

\author{
Carolina A.R. Funayama ${ }^{1}$; Marco Antônio Penna²; Marlene de Fátima Turcato²; \\ Carla Andrea T. Caldas ${ }^{3}$; Jucélia S. Santos ${ }^{3} \&$ Denise Moretto ${ }^{3}$
}

\begin{abstract}
${ }^{1}$ Docente. ${ }^{2}$ Médico Assistente do Hospital das Clínicas de Ribeirão Preto. ${ }^{3}$ Pós-graduando. Departamento de Neurologia, Psiquiatria e Psicologia Médica. Faculdade de Medicina de Ribeirão Preto-USP.

Correspondência: Profa. Dra. Carolina A. R. Funayama. Depto de Neurologia, Psiquiatria e Psicologia Médica. Faculdade de Medicina de Ribeirão Preto-USP. Av. Bandeirantes, 3900. CEP 14049-900 Ribeirão Preto, São Paulo, Brasil. e-mail: carfunay@fmrp.usp.br
\end{abstract}

FUNAYAMA CAR; PENNA MA; TURCATO MF; CALDAS CAT; SANTOS JS \& MORETTO D. Paralisia cerebral diagnóstico etiológico. Medicina, Ribeirão Preto, 33: 155-160, abr./jun. 2000.

RESUMO: Em estudo retrospectivo foram analisados 35 prontuários, obtidos ao acaso, de crianças com diagnóstico de Paralisia Cerebral (PC), atendidas no Hospital das Clínicas de Ribeirão Preto, entre 1982 e 1998, com o objetivo de verificar fatores etiológicos. As infecções congênitas $(36 \%)$ e agressão hipóxico-isquêmica (28\%) foram os mais freqüentes fatores determinantes de PC entre 25 casos com etiologia definida. Estes achados podem ser utilizados para estratégias regionais de prevenção de deficiências. Para os casos sem etiologia definida, lembram-se as formas herdadas de paralisia cerebral e o diagnóstico diferencial com doenças degenerativas.

UNITERMOS: Paralisia Cerebral. Infecção. Anoxia Recem-nascido; congênita; diagnóstico; etiologia.

\section{1- INTRODUÇÃO}

Paralisia Cerebral (PC) é uma expressão criada por Little $(1861)^{(1)}$, médico ortopedista, inglês. Little referia-se à paralisia (ausência de movimento) que ele diagnosticava nos membros, consideramdo a hipótese de ser ela decorrente de problemas cerebrais ocorridos durante o parto. Ao longo do século XX, os problemas obstétricos continuaram sendo preocupantes e foram minuciosamente estudados quanto à fisiopatogenia das lesões cerebrais deles decorrentes e ao seu valor prognóstico para seqüelas neurológicas ${ }^{(2,3)}$. Com o aperfeiçoamento das técnicas de ultra-sonografia, o advento da tomografia computadorizada (TC), ressonância magnética (RM), e com os avanços na assistência pré-natal, as malformações cerebrais, infecções maternas, congênitas e amnióticas, passaram a se destacar entre as principais causas de PC ${ }^{(4,5)}$ (Figura 1).
PC é definida como um distúrbio do movimento ou da postura decorrente de lesão cerebral não progressiva, que ocorre durante o período do desenvolvimento cerebral, limitando-se à infância ${ }^{(6)}$. Sua classificação foi primeiro proposta por Courville \& Marsh, $1944^{(7)}$, e vem se aperfeiçoando, com a proposta de Levitt, $1977^{(8)}$, classificando-a segundo o tipo e topografia da lesão cerebral (Tabela I). Freqüentemente observamos sinais de um e outro tipo no mesmo paciente, classificando-a como PC misto. Um tipo de PC hipotônico ou atônico foi introduzido na classificação pelo Little $\mathrm{Club}^{(6)}$. Entretanto, não se tem encorajado o seu uso: a hipotonia em membros, na criança, pode ser a fase pré-espástica da $\mathrm{PC}$ do tipo espástico ou o tono de repouso da PC com forma discinética. Além disso, há um amplo diagnóstico diferencial com a síndrome do bebê hipotônico, que tem, como causas mais frequientes, as doenças neuromusculares. 


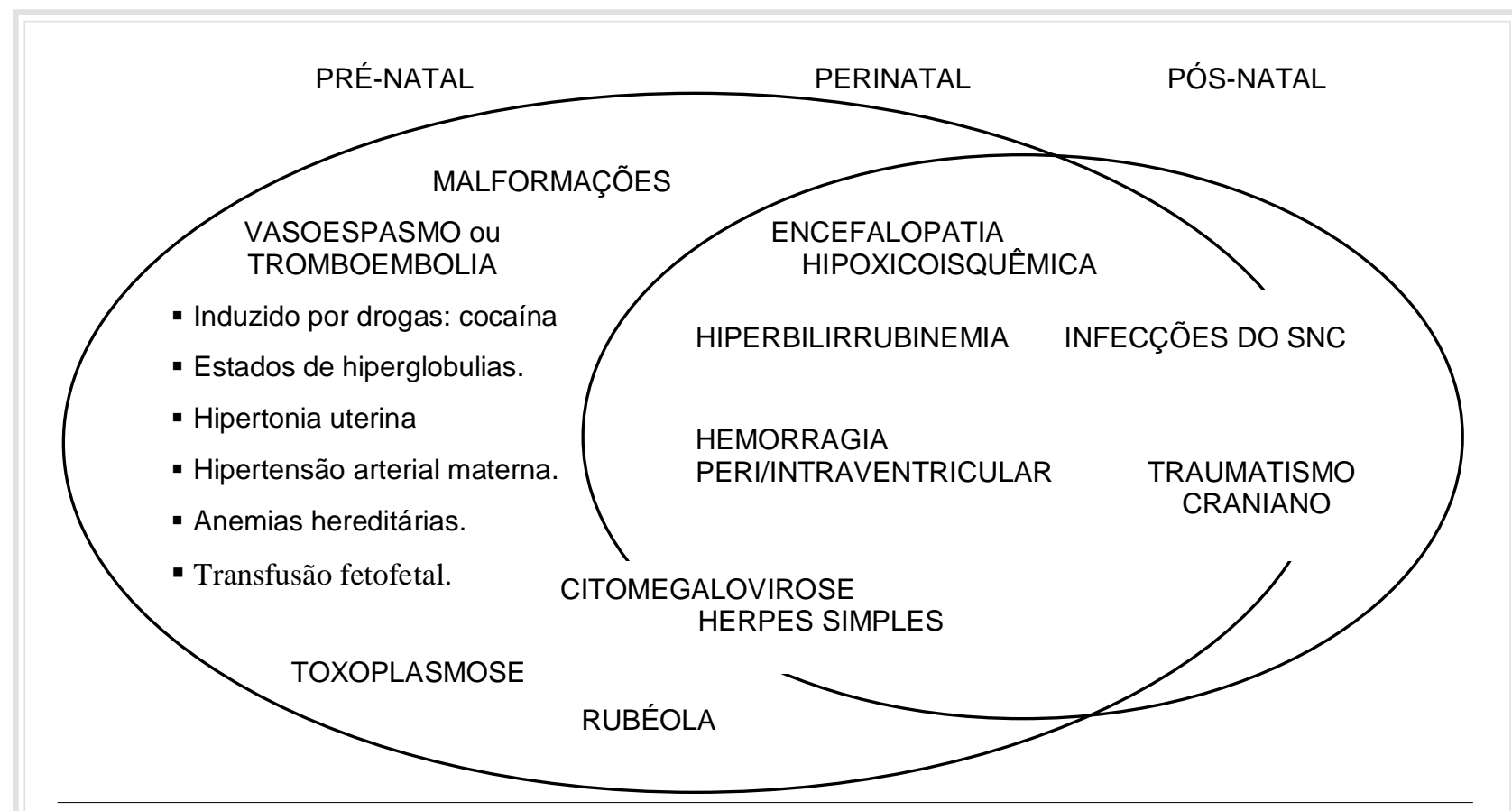

Figura 1: Etiologias mais comuns da paralisia cerebral.

\begin{tabular}{|lc|}
\hline \multicolumn{2}{|l|}{ Tabela I - Classificação mais conhecida da PC } \\
\hline Classificação topográfica & Classificação semiológica \\
\hline Piramidal & Espástica \\
\hline Extrapiramidal & Discinética: \\
& atetósica \\
& coreoatetósica \\
& distônica \\
\hline Cerebelar & Atáxica \\
\hline
\end{tabular}

\section{2- OBJETIVO}

Identificar as causas possíveis de PC em uma amostra de crianças atendidas em determinado hospital universitário, o Hospital das Clínicas de Ribeirão Preto-HCRP

\section{3- MATERIAL E MÉTODOS}

Aleatoriamente, entre os casos atendidos no Ambulatório de Neurologia Infantil do HCRP foram colhidos 35 registros de casos novos, diagnosticados como Paralisia Cerebral, atendidos entre os anos de 1986 e 1998. Desde 1982, um protocolo de atendimento para esse ambulatório vem contribuindo para a coleta de dados para fins de pesquisa clínica em neurodesenvolvimento. O diagnóstico etiológico foi revisto, caso a caso, verificando-se a história clínica e exames complementares.

\section{4- RESULTADOS}

Das 35 crianças, PC com forma tetraespástica foi diagnosticada em 22; diplégica, em três $(8,6 \%)$, paraparética, em dois $(5,7 \%)$; hemiparética em três $(8,6 \%)$; forma mista, em três $(8,6 \%)$ e discinética, em uma $(2,8 \%)$.

As causas foram definidas em 25 casos (Tabela II). Os 10 restantes foram considerados como PC provável, por apresentarem quadro estático, porém sem causa definida (Tabela III).

Das 35 crianças, quatro foram acompanhadas desde o período neonatal: uma, com diagnóstico de toxoplasmose congênita, nasceu no HCRP e, com um mês de idade, já apresentava tetraespasticidade. Três outras, encaminhadas aos três, quatro e seis dias de vida, com quadro agudo de intoxicação bilirrubínica, foram submetidas à exsangüineotransfusão: uma delas apresentou tétano neonatal e evoluiu com tetraespasticidade no primeiro mês. Duas outras tiveram reação de inibição da hemaglutinação para Rubéola 1/ 16 e 1/320, respectivamente, sem sinais clínicos de infecção congênita. Esses títulos foram mantidos em uma segunda e última amostra, deixando dúvidas 


\begin{tabular}{|c|c|c|}
\hline \multicolumn{3}{|l|}{ Tabela II - Causas de PC em 25 crianças } \\
\hline Causas da paralisia cerebral & $N$ & $\%$ \\
\hline \multicolumn{3}{|l|}{ Fatores pré-natais } \\
\hline - Lissencefalia tipo I (S. Miller-Dieker) & 1 & 4 \\
\hline - Hidranencefalia & 1 & 4 \\
\hline $\begin{array}{l}\text { - Esquizencefalia (lábio aberto, } \\
\text { bilateral, simétrico) }\end{array}$ & 1 & 4 \\
\hline - Infecção congênita & 9 & 36 \\
\hline \multicolumn{3}{|l|}{ Fatores perinatais } \\
\hline - Leucomalácia periventricular & 3 & 12 \\
\hline $\begin{array}{l}\text { - Hemorragia intraventricular com } \\
\text { hidrocefalia }\end{array}$ & 1 & 4 \\
\hline $\begin{array}{l}\text { - Encefalopatia hipoxicoisquêmica ao } \\
\text { nascimento }\end{array}$ & 4 & 16 \\
\hline - Hiperbilirrubinemia + infecção (?) & 2 & 8 \\
\hline - Hiperbilirrubinemia + tétano neonatal & 1 & 4 \\
\hline \multicolumn{3}{|l|}{ Fatores pós-natais } \\
\hline - Meningite meningocócica & 1 & 4 \\
\hline - Traumatismo craniano & 1 & 4 \\
\hline Total & 21 & 100 \\
\hline
\end{tabular}

quanto à infecção congênita; uma evoluiu com hipotonia global, seguida de espasticidade em membros inferiores e, aos 10 meses, foram observados movimentos coreoatetósicos, configurando, portanto, uma forma mista de PC; a outra evoluiu com tetraespasticidade, diagnosticada ainda no segundo mês.

A idade das demais crianças no primeiro atendimento, encaminhadas de outros serviços para o Ambulatório de Neurologia Infantil, variou de cinco meses a 12 anos, mediana de 21 meses.

As causas mais frequientes de PC entre as 25 com diagnóstico definido foram as infecções congênitas (36\%) e agressão hipoxicoisquêmica (28\%). Das infecções congênitas, o diagnóstico foi definido, sorologicamente, em três casos, dois com toxoplasmose e um com citomegalovirose; nos seis outros, a infecção congênita foi considerada altamente provável, considerando-se o quadro clínico e de imagem cerebral: duas com a tríade clássica da toxoplasmose: hidrocefalia, calcificações intracerebrais e coriorretinite, uma com sinais típicos da rubéola congênita: surdez, microcefalia, catarata e cardiopatia, e as demais, indistingüíveis, se toxoplasmose ou citomegalovirose: microcefalia e calcificações intracranianas, hidrocefalia acentuada por atrofia.
Em relação às lesões hipoxicoisquêmicas, o diagnóstico, em três bebês nascidos pré-termo, baseouse, exclusivamente, nos achados de imagem: a leucomalácia periventricular, em dois, e discreta atrofia cortical, com hiperdensidade talâmica, em um. Para quatro crianças nascidas a termo, o diagnóstico de EHI foi definido, considerando-se a presença dos seguintes dados: história clínica de fatores de risco para sofrimento fetal, informações de hipoatividade reflexa neonatal (reflexos primitivos, como a sucção, por exemplo) somados aos achados sugestivos de atrofia cortical em imagem cerebral por TC ou RM.

O diagnóstico etiológico dos demais pacientes, apresentados na Tabela II, foi concluído por exames de imagem: TC ou RM.

\section{5- DISCUSSÃO}

Os achados do presente trabalho nos indicam as dificuldades para diagnosticar, retrospectivamente, causas pré e perinatais de PC.

Considerando os casos aqui apresentados com diagnóstico etiológico definido, verificamos o predomínio dos fatores pré-natais. A lissencefalia do tipo I, como a Síndrome de Miller-Dieker (Figura 2), aqui diagnosticada, é um defeito no período da migração neuronal, que ocorre entre 10 e 20 semanas gestacionais. Em vários indivíduos com Síndrome de MillerDieker, foi verificada microdeleção, envolvendo o $17 \mathrm{p} 13.3^{(9)}$. A hidranencefalia, na maioria das vezes, é decorrente de bloqueio das carótidas por prováveis fatores trombóticos ou embólicos num período precoce da gravidez ${ }^{(10)} \mathrm{e}$, raras vezes, é familial, com risco de recorrência ${ }^{(11)}$. A esquizencefalia, outrora vista como um porencéfalo que se comunica com os ventrículos cerebrais, é, hoje, considerada como patologia distinta, resultante de mutação gênica ${ }^{(12)}$.

A inclusão dos casos de infecção congênita provável, no grupo com PC definida, se justifica, dada a extrema raridade de outras doenças que cursam com quadro semelhante ${ }^{(13)}$.

A baixa freqüência de casos com seqüela de lesões hipoxicoisquêmicas, nas crianças nascidas a termo, justifica-se na presente casuística, seja pelo baixo número de casos, encaminhados de outros serviços, que consideram o diagnóstico já realizado e, portanto, não necessitando de mais investigação, seja considerando-se, também, pela criteriosa avaliação dos casos com definição da encefalopatia hipoxicoisquêmica perinatal como elemento principal para o diagnóstico da PC. Há muito, a literatura médica vem reafirmando que, em caso de sofrimento fetal por hipoxia ou 


\begin{tabular}{|c|c|c|c|c|}
\hline $\begin{array}{l}\text { Ano } \\
\text { nasc. }\end{array}$ & $\begin{array}{l}\text { Gestação } \\
\text { duração }\end{array}$ & $\begin{array}{l}\text { Idade no } 1^{\circ} \\
\text { atendimento }\end{array}$ & PC & Dados para discussão sobre fatores causais \\
\hline 1981 & termo & 8anos & $\begin{array}{l}\text { tetraparética } \\
\text { espástica }\end{array}$ & $\begin{array}{l}\text { Pré-natal sem intercorrências. } \\
\text { Demorou para chorar. Parto domiciliar. } \\
\text { Sem exames complementares. }\end{array}$ \\
\hline 1984 & termo & 12 anos & $\begin{array}{l}\text { hemiparética } \\
\text { espástica }\end{array}$ & $\begin{array}{l}\text { Pré e perinatal sem intercorrências. } \\
\text { IRM de cranioinfarto antigo, parietal esquerdo }\end{array}$ \\
\hline 1988 & pré-termo & 3 anos & $\begin{array}{l}\text { diplegia } \\
\text { espástica }\end{array}$ & $\begin{array}{l}\text { Pré-natal sem intercorrências. } \\
29 \text { dias em incubadora. } \\
\text { Sem exames complementares. }\end{array}$ \\
\hline 1988 & termo & 9 meses & $\begin{array}{l}\text { tetraparética } \\
\text { espástica }\end{array}$ & $\begin{array}{l}\text { Pré e perinatal sem intercorrências. } \\
\text { TC de crânio - atrofia focal parietal direita. }\end{array}$ \\
\hline 1992 & termo & 2 anos & $\begin{array}{l}\text { hemiparética } \\
\text { espástico }\end{array}$ & $\begin{array}{l}\text { Pré e perinatal sem intercorrências. } \\
\text { TC de crânioinfarto antigo, parietal direito. }\end{array}$ \\
\hline 1994 & termo & 9 meses & $\begin{array}{l}\text { tetraparética } \\
\text { espástica }\end{array}$ & $\begin{array}{l}\text { Pré-natal sem intercorrências. } \\
\text { CMV- IgG } 1 / 64 \text {. }\end{array}$ \\
\hline 1996 & gemelar & $\begin{array}{l}1 \text { ano e } \\
5 \text { meses }\end{array}$ & $\begin{array}{l}\text { tetraparética } \\
\text { espástica }\end{array}$ & $\begin{array}{l}\text { Pré-natal sem intercorrências. } \\
\text { CMV -lgG 1/64 }\end{array}$ \\
\hline 1996 & $\begin{array}{l}\text { Pré- } \\
\text { termo }\end{array}$ & $\begin{array}{l}1 \text { ano e } \\
9 \text { meses }\end{array}$ & $\begin{array}{l}\text { paraparética } \\
\text { espástica }\end{array}$ & $\begin{array}{l}\text { Pré-natal sem intercorrências. } \\
\text { Alta após três dias no berçário. } \\
\text { TC de crânio: normal }\end{array}$ \\
\hline 1996 & Pré-termo & $\begin{array}{l}1 \text { ano e } \\
8 \text { meses }\end{array}$ & $\begin{array}{l}\text { mista } \\
\text { tetraparesia } \\
\text { espástica e } \\
\text { distônica }\end{array}$ & $\begin{array}{l}\text { Pré e perinatal sem intercorrências. } \\
\text { IRM- hipossinal em putâmen à esquerda, } \\
\text { que pode corresponder à calcificação. } \\
\text { TC- calcificações em núcleos da base. }\end{array}$ \\
\hline 1996 & termo & 9 meses & $\begin{array}{l}\text { mista } \\
\text { monoparesia } \\
\text { espástica e } \\
\text { atáxica }\end{array}$ & $\begin{array}{l}\text { Pré e perinatal sem intercorrências. } \\
\text { Rubéola - R. hemaglutinação 1/8 } \\
\text { IRM de crânio - normal }\end{array}$ \\
\hline \multicolumn{3}{|c|}{ IRM - Imagem por Ressonância Magnética } & \multicolumn{2}{|c|}{ TC - Tomografia computadorizada } \\
\hline
\end{tabular}

isquemia periparto, esses são os achados neurológicos no neonato que apresentam maior sensibilidade como fator prognóstico ${ }^{(14)}$.

As crianças com antecedente de hiperbilirrubinemia, na presente amostra, podem ter tido infecções associadas. Reafirma-se com estes relatos a constatação $^{(15)}$ de que as infecções são agravantes dos fatores de risco de PC, como hipoxia e isquemia, ressaltando-se o enfoque às corioamnionites, que, além do alto risco de contaminação fetal, induzem ao parto prematuro $^{(11)}$. Chamamos a atenção para o fato de que, das três crianças com kernicterus, apenas uma apresentou a forma de PC distônica pura. Isso significa que, nas demais crianças, a lesão pode ter predominado nos núcleos basais, embora outras regiões também tenham sido atingidas, como o córtex, que, muitas vezes, se atrofia; ou ainda, podem ter ocorrido causas concorrentes, como hipoxia e infecções.
Três casos com suspeita de infarto cerebral antigo e, provavelmente, pré-natal não foram incluídos entre aqueles com causa definida, porque, neles, subentende-se um acidente vascular cerebral precoce, de causa a ser esclarecida. Esses infartos podem ser decorrentes de distúrbios sistêmicos maternos, como anemia, trauma, envenenamentos, coagulopatias e abuso de cocaína, ou condições fetais como gemelaridade, hidropsia, discrasia sangüínea, por exemplo ${ }^{(10)}$. Tais causas permitem o enquadramento como causas de PC. Por outro lado, podemos considerar hipóteses etiológicas, como a homocistinúria, deficiência de G6P ou as mitocondriopatias com manifestações isquêmicas ${ }^{(16)}$, entre outras doenças degenerativas que, cursando com deterioração progressiva, não podem ser consideradas causas de PC.

Em outra criança com PC provável, havia sinais tomográficos sugestivos de calcificações em nú- 


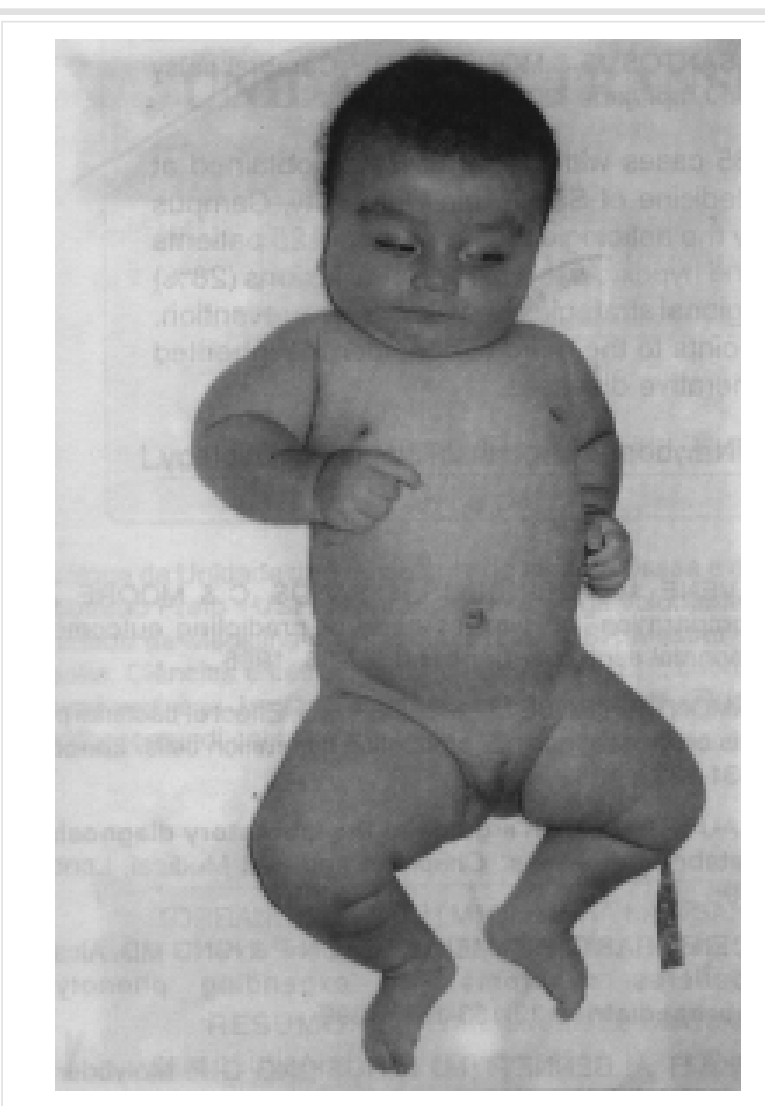

Figura 2 - Criança com lissencefalia tipo I (Síndrome de Miller-Dieker) aos 8 meses de idade, em fase de transição de hipotonia para espasticidade. Observar a fronte proeminente, raiz nasal baixa, narinas antevertidas, lábios finos, e ângulo aberto dos adutores das coxas (atitude de batráquio - sinal de hipotonia).

cleos basais, raramente decorrentes de lesão hipoxicoisquêmica e comuns a uma série de doenças tóxicas, infecciosas e degenerativas ${ }^{(17)}$.

Há alguns erros inatos do metabolismo, que são doenças degenerativas, hereditárias, com repercussão grave para o sistema nervoso, que se apresentam com quadro muito semelhante ao da PC tetraespástica ou discinética. São exemplos a deficiência da sulfitoxidase, ou do seu cofator, molibdênio, que podem se manifestar com tetraespasticidade precoce, crises convulsivas e imagem sugestiva de encefalomalácia multicística $^{(18,19)}$.

Na PC, a instalação do quadro espástico, nos membros, ocorre após um período curto de hipotonia, de modo geral entre um e três meses, havendo, às vezes, período mais prolongado em caso de malformações como na Síndrome de Miller-Dieker. Entretanto, um período variável de hipotonia até instalar-se a espasticidade também pode ocorrer em doenças degenerativas, como na doença de Krabbe ${ }^{(20)}$ (deficiência de galactocerebrosidase), ou erros ligados a oligoelementos, como na Síndrome de Menkes ${ }^{(21)}$ (baixa absorção de cobre).

Também devemos estar atentos para o diagnóstico diferencial das PC discinéticas com doenças degenerativas, principalmente as mitocondriopatias e acidúrias orgânicas ${ }^{(22)}$, nas quais a distonia pode se iniciar a qualquer momento, na infância. $\mathrm{Na} \mathrm{PC}$ de forma discinética, a instalação do quadro distônico ou coreoatetósico, após a lesão cerebral, também pode se iniciar a qualquer momento na infância, mas em até três anos na maioria dos $\operatorname{casos}^{(23)}$.

A forma diplégica ou a paraparética espástica está mais freqüentemente associada à prematuridade, porque o cérebro imaturo sofre os efeitos das condições adversas fora do útero, como dificuldades respiratórias ou de manutenção da pressão arterial, levando a hiper ou hipofluxo cerebral e ocasionando danos teciduais do tipo leucomalácia periventricular, hemorragia perintraventricular ou intraparenquimatosa ${ }^{(3)}$. Entretanto, chama atenção a freqüência com que se encontram crianças paraparéticas ou mesmo plégicas espásticas com exames de imagem cerebrais normais. Nesses casos, devemos lembrar a possibilidade de lesões hipoxicoisquêmicas ou traumáticas medulares perinatais ${ }^{(24)}$, que são de difícil diagnóstico. Impõe-se, ainda, o diagnóstico diferencial com doenças degenerativas do SNC, algumas com progressão muito lenta, como a paraparesia espástica familial, com início na infância e que pode se acompanhar de alterações cerebelares $^{(25)}$.

Resumindo, uma vez diante de uma criança com sinais de lesão piramidal, extrapiramidal ou cerebelar, para a identificação de fatores causais, é fundamental retomar toda a história pregressa, tendo à mão exames do período pré-natal e do período em que os eventos adversos ocorreram. $\mathrm{O}$ exame de escolha para auxílio na elucidação diagnóstica é a TC de crânio, porque ela nos fornece, com segurança, sinais de calcificações. Na seqüência da investigação, não sendo esclarecedora a TC, indica-se a RM, que pode apresentar sinais de defeitos do desenvolvimento neurológico, e, também, nos possibilita imagens de doenças degenerativas, como os erros inatos, e outras sem erro bioquímico definido. Em alguns casos de suspeita de PC, a presença de dismorfias, outros casos semelhantes na família, ausência de problemas no período pré e perinatal, são algumas situações para encaminhamento à avaliação genética. 
FUNAYAMA CAR; PENNA MA; TURCATO MF; CALDAS CAT; SANTOS JS \& MORETTO D. Cerebral palsy aetiologic diagnosis. Medicina, Ribeirão Preto, 33: 155-160, apr./june 2000.

ABSTRACT: A retrospective study was carried on 35 cases with Cerebral Palsy, obtained at random, attended at the Hospital of Clinics of School Medicine of São Paulo University, Campus Ribeirão Preto, from 1982 to 1998, with the scope to verify the aetiologic factors. Among 25 patients with identified aetiology, the Congenital infections (36\%) and hypoxic-schemic cerebral lesions (28\%) were the major causes. These results could be used for regional strategies of deficiencies prevention. Comments about the cases without aetiology identified points to the need to consider the inherited cerebral palsies and the differential diagnosis with degenerative diseases.

UNITERMS: Cerebral Palsy. Infection. Anoxia Infant, Newborn; congenital; diagnosis; etiology.

\section{REFERÊNCIAS BIBLIOGRÁFICAS}

1 - LITTLE WJ. On the influence of abnormal parturition difficult labour, premature birth, and asphyxia neonatorum on the mental and physical condition of the child. Lancet 2 : 378-380, 1861.

2 - COURVILLE C.B. Contributions to the study of the cerebral anóxia. San Lucas Press, Los Angeles, 1953.

3 - LEVENE MI. The asphyxiated newborn infant. In: LEVENE MI \& LILFORD RJ, eds. Fetal and neonatal neurology and neurosurgery. $2^{\text {nd }}$ ed. Churchill Livingstone, New York, p. 405-425, 1995

4 - JAW TS; SHEU RS; LIU GC \& CHOU MS. Magnetic resonance images of neuronal migration anomalies. Kao Hsiung I Hsueh Ko Hsueh Tsa Chih, 14: 504-513, 1998

5 - GRETHER JK \& NELSON KB. Maternal infection and cerebral palsy in infants of normal birth weight. JAMA 278: 207-211, 1997

6 - LITTLE CLUB CLINICS IN DEVELOPMENTAL MEDICINE. William Heinemann Medical Books, London, 1960.

7 - COURVILLE CB \& MARSH C. Neonatal asphyxia. Its encephalic residuals and the mechanism of their production. Bull Los Angeles Neurol Soc 9:121-134,1944

8 - LEVITT S. Treatment of cerebral palsy and motor delay. Blackwell Scientific Publications, Oxford, 1977.

9 - DOBYNS WB; CURRY CJR; HOYME HE; TURLINGTON L \& LEDBETTER DH. Clinical and molecular diagnosis of MillerDieker syndrome. Am J Hum Genet 48:584-594,1991

10 - LARROCHE JC. Fetal cerebral pathology of circulatory origin. In: LEVENE MI \& LILFORD RJ, eds. Fetal and neonatal neurology and neurosurgery. $2^{\text {nd }}$. ed. Churchill Livingstone, New York, p. 322-322, 1995

11 - HAMBY WB; KRAUSS RF \& BESWICK WF. Hydranencephaly: clinical diagnosis. Presentation of seven cases. Pediatrics 6:371-383,1950

12 - FAIELLAA; BRUNELLI S; GRANATA T; DINCERTI L; CARDINI R; LENTI C; BATTAGLIA G \& BONCINELLI E. A number of schizencephaly patients including 2 brothers are heterozygous for germline mutations in the homeobox gene EMX2. Eur J Hum Genet 5:186-190, 1997

13 - De ROSSO AL; MARANHÃO FILHO P DE A; DE OLIVEIRA EA \& NOVIS SA. Diffuse encephalic calcification. A case report. Arq Neuropsiquiatr 50: 519-522, 1992
14 - LEVENE MI, GRINDULIS H, SANDS C \& MOORE J R. Comparation of two methods of predicting outcome in perinatal asphyxia. Lancet II: 67-68, 1986.

15 - LAMONT RF; ROSE MP \& ELDER MG. Effect of bacterial products on prostaglandin $\mathrm{E}$ production by amnion cells. Lancet II: 1331-1333, 1985

16 - BLAU N. Physician's guide to the laboratory diagnosis of metabolic diseases. Chapman and Hall Medical, London, 1996

17 - MCENTAGART M; KAMEL H; LEBON P \& KING MD. AicardiGoutières syndrome: an expanding phenotype. Neuropediatrics 29:163-167, 1998

18 - AUKETT A; BENNETT MJ \& HOSKING G P. Molybdenum co-factor deficiency: an easily missed inborn error of metabolism. Dev Med Child Neurol 30:531-535, 1988.

19 - SCHUIERER G; KURLEMANN G; BICK U \& STEPHANI U. Molybdenum cofactor deficiency: CT and MR findings. Neuropediatrics 26:51-54, 1995

20 - KRABBE K. A new familial, infantile form of diffuse brain sclerosis. Brain 39: 74- 114, 1916

21 - MENKES JH, ALTER M, STEIGLEDER GK, WEAKLEY DR \& SUNG JH. A sex-linked recessive disorder with growth retardation, peculiar hair and focal cerebral and cerebellar degeneration. Pediatrics 29:764-779, 1962

22 - CHAMOLES N; GALICCHIO S; GRAGNANA N; LAFUENTEA; JORGE L \& FUSTAM. Acidurias orgánicas: prevalencia de movimientos anormales. Rev Neurol (Esp) 27: 324,1998 Resumo.

23.BURKE RE; FAHN S \& GOLD AP. Delayed-onset dystonia in patients with "static" encephalopathy. J Neurol Neurosurg Psychiatr 43:789-797, 1980

24 - CLANCY RR; SLADKY JT \& RORKE LB. Hypoxi-schemic spinal cord injury following perinatal asphyxia. Ann Neurol 25:185-189,1989

25 - HARDING AE. Hereditary 'pure' spastic paraplegia: a clinical and genetic study of 22 families. J Neurol Neurosurg Psychiatr 44:871-883,1981

Recebido para publicação em 10/01/2000

Aprovado para publicação em 06/04/2000 